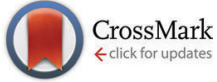

Cite this: Phys. Chem. Chem. Phys., $2015,17,11954$

Received 4th March 2015

Accepted 6th April 2015

DOI: $10.1039 / c 5 c p 01283 j$

www.rsc.org/pccp

\section{Surface enhanced resonance Raman detection of a catalytic intermediate of DyP-type peroxidase $\uparrow$}

\author{
Smilja Todorovic, ${ }^{{ }^{a}}$ Peter Hildebrandt ${ }^{\mathrm{b}}$ and Lígia O. Martins ${ }^{\mathrm{a}}$
}

\begin{abstract}
We report herein the vibrational spectroscopic characterisation of a catalytic intermediate formed by the reaction of $\mathrm{H}_{2} \mathrm{O}_{2}$ with DyP-type peroxidase immobilised on a biocompatible coated metal support. The SERR spectroscopic approach is of general applicability to other peroxidases which form relatively stable catalytic intermediates.
\end{abstract}

Dye-decolourising peroxidases (DyPs) are novel heme peroxidases, the primary sequence, structural and mechanistic properties of which are unrelated to those of other known peroxidases. ${ }^{1-10}$ They lack the distal histidine that is highly conserved in classical peroxidases and which acts as an acid/base catalyst in the reduction of hydrogen peroxide to water. DyPs are classified into four phylogenetically distinct classes (A-D); their physiological role is not fully established yet and it appears to be subfamilydependent..$^{1-10}$ DyP from Pseudomonas putida MET94 (PpDyP) is an extremely versatile B-type DyP, capable of efficient oxidation of a wide range of anthraquinonic and azo dyes, phenolic substrates, the non-phenolic veratryl alcohol and even manganese and ferrous ions. ${ }^{8}$ In the reaction with $\mathrm{H}_{2} \mathrm{O}_{2}$, PpDyP forms a stable Compound I (Cpd I) at a rate of $1.4 \pm 0.3 \times 10^{6} \mathrm{M}^{-1} \mathrm{~s}^{-1}$, comparable to those of classical peroxidases and other DyPs. ${ }^{8}$ The intermediate is, like in other DyPs, surprisingly long-living, with a half-life of $\sim 60 \mathrm{~min}$, as demonstrated by electronic absorption spectroscopy. ${ }^{9}$ Upon immobilisation on biocompatible metal supports, PpDyP is capable of efficient electrocatalytic activity in the presence of hydrogen peroxide. The enzyme is therefore a promising candidate for the design of bio-electronic devices with unique DyP-type peroxidase substrate specificity. ${ }^{9,10}$ Resonance Raman (RR) spectroscopy has been extensively used in the detection and characterisation of peroxidase and catalase-peroxidase catalytic intermediates, and

\footnotetext{
${ }^{a}$ Instituto de Tecnologia Química e Biológica (ITQB), Universidade Nova de Lisboa, Av.da República,2780-157 Oeiras, Portugal.E-mail: smilja@itqb.unl.pt

${ }^{b}$ Institut für Chemie, Sektr. PC14, Technische Universität Berlin,

Strasse des 17. Juni 135, D-10623 Berlin, Germany

$\dagger$ Electronic supplementary information (ESI) available: Experimental details of protein purification and spectroscopic experiments, together with SERR spectra of the immobilised PpDyP upon addition of $\mathrm{H}_{2} \mathrm{O}_{2}$ in the $600-1700 \mathrm{~cm}^{-1}$ range. See DOI: $10.1039 / \mathrm{c} 5 \mathrm{cp} 01283 \mathrm{j}$
}

RR spectroscopic fingerprints of Cpd I (two equivalent oxidised resting ferric state) and Cpd II (one equivalent oxidised resting ferric state) are well established. ${ }^{11-16}$ However, a strong fluorescence impeded the RR studies of PpDyP intermediates. Here, we have probed catalytic intermediate species of immobilised PpDyP by surface enhanced RR (SERR) spectroscopy. The SERR spectra of heme proteins immobilised on nanostructured Ag surfaces exclusively display the cofactor signals of the adsorbed molecules. In addition, close proximity of the molecule to the metal surface can efficiently quench fluorescence. ${ }^{17}$ Like in RR spectroscopy, SERR signals include, inter alia, the core-size marker bands (e.g. $\left.\nu_{4}, \nu_{3}, \nu_{2}, \nu_{10}\right)$ which are specifically enhanced upon excitation in resonance with the Soret absorption band of the porphyrin. The frequencies of these modes are indicative of the redox and spin states and the coordination pattern of the heme iron.

We have previously shown that the high frequency region of RR spectra of the resting PpDyP in solution displays broad marker bands, indicative of the co-existence of several spin populations. ${ }^{9,10}$ Component analysis of the spectra reveals well resolved bands of the $\nu_{4}, \nu_{3}, \nu_{2}, \nu_{10}$ and $\nu_{\mathrm{C}=\mathrm{C}}$ modes at 1376, 1502, 1572, 1626 and $1637 \mathrm{~cm}^{-1}$ (Fig. 1a, green trace) and at 1373, 1494, 1564, 1621 and $1632 \mathrm{~cm}^{-1}$ (Fig. 1a, blue trace), which are attributed to quantum mechanically mixed-spin (QS) and 5-coordinated high spin (5cHS) species, respectively. ${ }^{9,10}$ In addition, small contributions of a 6-coordinated HS $(6 \mathrm{cHS})$ species with $\nu_{4}$ at $1366 \mathrm{~cm}^{-1}$ and $\nu_{3}$ at $1483 \mathrm{~cm}^{-1}$ are also present in the spectra. The SERR spectra of PpDyP immobilised on Ag supports coated with mixed amino- and hydroxyl-terminated alkanethiol self-assembled monolayers (SAM), employing aminooctanethiol (AOT) and mercaptohexanol (MOH) in a $1: 3(\mathrm{M} / \mathrm{M})$ ratio, reveal essentially the same band frequencies as RR spectra (Fig. 1a and b). This indicates that the protein structure is preserved, on the level of the heme group, upon adsorption. We observe a slight alteration of relative contributions of QS and $5 \mathrm{cHS}$. The former species, which is dominant in solution, appears to be less abundant in the immobilised state. This finding is related to a highly flexible heme cavity observed in 


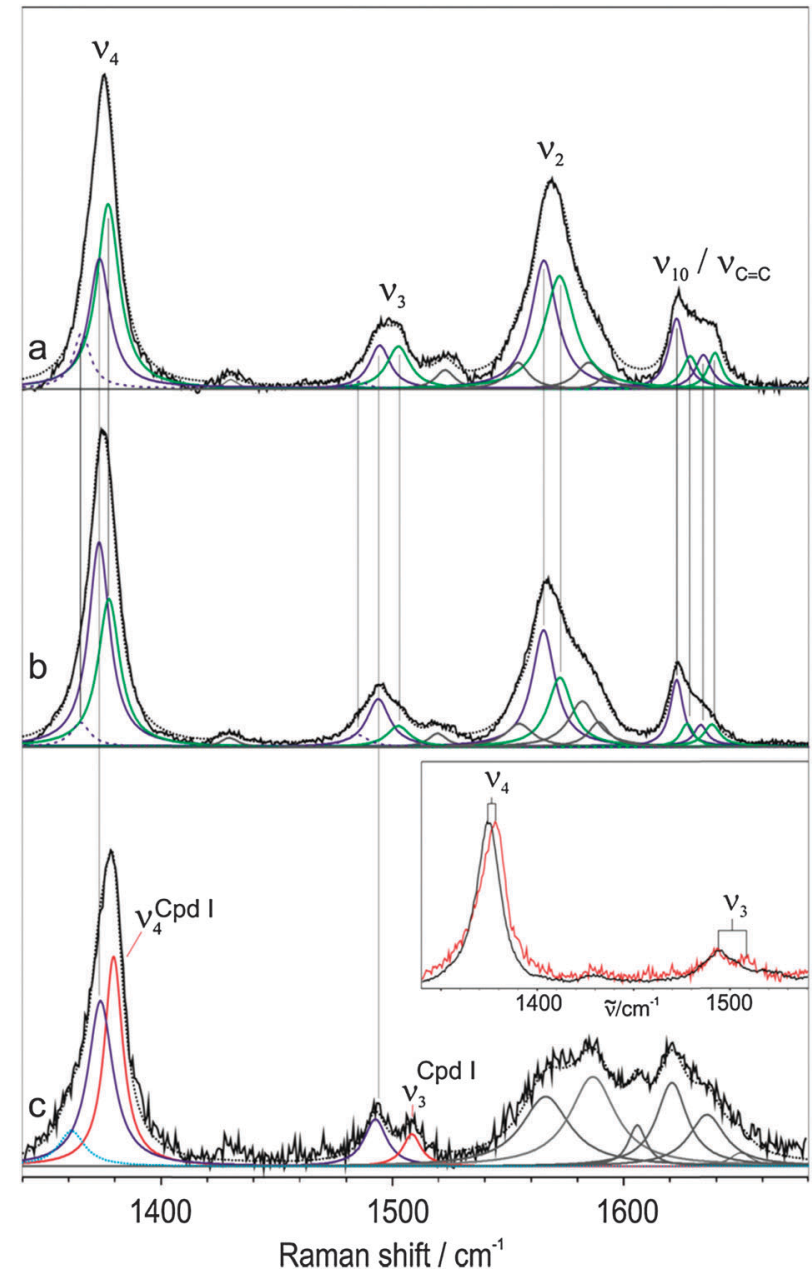

Fig. 1 High frequency region of the RR and SERR spectra of ferric PpDyP in the absence/presence of hydrogen peroxide. Experimental and component RR spectra of PpDyP (a), SERR spectra of PpDyP immobilised on AOT/MOH SAM in the absence (b) and presence of 1 equivalent of $\mathrm{H}_{2} \mathrm{O}_{2}$ (c). Inset: SERR spectra of the immobilised resting state PpDyP (black trace) and Cpd I (red trace) in the $\nu_{4} / \nu_{3}$ mode region. After polynomial baseline subtraction, the experimental spectra (black trace) were subjected to component analysis. ${ }^{9}$ The fits (dotted black trace) are based on the component spectra of $6 \mathrm{cHS}$ (blue dashed trace), $5 \mathrm{cHS}$ (blue solid trace) and QS (green trace) species (a and b), compound I (red trace) and photoreduced (turquoise trace) species (c), together with non-assigned and/or spin state insensitive bands (grey trace). RR and SERR spectra were recorded with $413 \mathrm{~nm}$ excitation, 5 and $1.1 \mathrm{~mW}$ laser power and 60 and $20 \mathrm{~s}$ accumulation time, respectively; SERR spectra were recorded at the open circuit. RR experiments were carried out with $100 \mu \mathrm{M}$ (SERR with $0.4 \mu \mathrm{M})$ protein in buffered solution $(50 \mathrm{mM}$ Tris- $\mathrm{HCl}+200 \mathrm{mM} \mathrm{NaCl}$, $\mathrm{pH}$ 7.6). Two RR spectra (a) and four (b) or eight (c) SERR spectra were co-added to increase $S / N$. Note that no assignments are given for the bands in the $\nu_{2} / \nu_{10}$ envelope (c) due to the lack of reference RR data on catalytic intermediates of DyPs.

DyPs (and other bacterial and plant peroxidases) in which the temperature, $\mathrm{pH}$ and physical state of the enzyme (i.e. solution $v s$. crystalline form) induce a typically reversible inter-conversion of the spin populations. ${ }^{9,10,18,19}$

Upon addition of 1 equivalent of hydrogen peroxide to the SERR cell holding the enzyme-loaded metal support, well reproducible spectral changes occur instantaneously: a pronounced decrease of signal intensity, accompanied by an upshift of $\nu_{4}$ and $\nu_{3}$ modes and a broadening of $\nu_{2}, \nu_{10}$ and $\nu_{\mathrm{C}=\mathrm{C}}$ bands (Fig. S1, ESI $\dagger$ ). Component analysis of the spectra suggests an equilibrium of two predominant species, one of which is characterised by $\nu_{4}$ and $\nu_{3}$ at 1373 and $1494 \mathrm{~cm}^{-1}$ and the other newly formed one with $\nu_{4}$ and $\nu_{3}$ at 1378 and $1508 \mathrm{~cm}^{-1}$ (Fig. 1c, blue and red trace, respectively). We attribute the former population to the $5 \mathrm{cHS}$ (vide supra) and the latter to the Cpd I - like species. This assignment is consistent with the frequencies reported in the literature for catalytic intermediates of classical peroxidases. ${ }^{11-16}$ In the absence of the reference RR spectra of Cpd I and Cpd II in DyPs, the assignment of the $1378 \mathrm{~cm}^{-1}$ and $1508 \mathrm{~cm}^{-1}$ modes to the oxyferryl $\mathrm{Fe}(\mathrm{Iv})=\mathrm{O}$ porphyrin $\pi$ cation is supported by the evidence from our previous work: (i) electronic absorption spectra, which reveal a fingerprint of stable Cpd I in the reaction of PpDyP with 1-3 equivalents of $\mathrm{H}_{2} \mathrm{O}_{2}$ in solution; $;^{8-10}$ and (ii) electrochemical data which demonstrate that PpDyP immobilised on AOT/MOH SAMs is electrocatalytically active and capable of $\mathrm{H}_{2} \mathrm{O}_{2}$ reduction. ${ }^{9}$ We can therefore rule out the possibility that inactive $\mathrm{Cpd}$ III ( $\mathrm{Fe}(\mathrm{III})-\mathrm{O}_{2}{ }^{-}$or $\mathrm{Fe}(\mathrm{II})-\mathrm{O}_{2}$ ) is formed upon addition of $\mathrm{H}_{2} \mathrm{O}_{2}$ to the immobilised PpDyP. However, distinguishing between Cpd I and Cpd II is not straightforward at this point (note that some DyPs are, like lignin and versatile peroxidases, prone to spontaneous reduction of Cpd I to a Cpd II species). ${ }^{8}$ The $\nu_{4} / \nu_{3}$ values that we observe here are slightly higher than those reported for Cpd I in the literature, ${ }^{11}$ which may be an intrinsic property of DyPs, or an indication of Cpd II. For instance, $\nu_{4}$ and $\nu_{3}$ modes of Cpd I in HRP were found at 1373-1376 and $1505 \mathrm{~cm}^{-1}$, respectively, but at 1379 and $1510 \mathrm{~cm}^{-1}$ in Cpd II. ${ }^{14}$ Nevertheless, RR measurements of these intermediates in solution are in most of the cases accompanied by experimental difficulties that frequently lead to the formation of photoreduced species. ${ }^{14}$ Bands of these side products may partially mask those of the catalytic intermediates. We could actually identify traces of a population with $\nu_{4}$ at $1361 \mathrm{~cm}^{-1}$ in the SERR spectra of the immobilised PpDyP that most likely originates from a photoreduced Cpd I (Fig. 1c, turquoise trace). Additional support for the assignment of the 'new' species to Cpd I comes from the attenuated SERR signal intensity upon addition of $\mathrm{H}_{2} \mathrm{O}_{2}$ (Fig. S1, ESI $\dagger$ ). It is most likely related to a decrease and moderate blue shift of the Soret absorption band ( $\lambda_{\text {soret }}=405 \mathrm{~nm}$ for the resting state PpDyP and $401 \mathrm{~nm}$ for Cpd I), which are characteristic of $\mathrm{Cpd} \mathrm{I},{ }^{8-10}$ and may cause a distinct loss of resonance enhancement in the spectra upon excitation at $413 \mathrm{~nm}$ (note that partial desorption of the enzyme cannot be ruled out). Formation of Cpd II, on the other hand, causes a significant red-shift of the Soret band to $417 \mathrm{~nm},{ }^{8-10}$ without pronounced intensity changes, which should actually have an opposite effect on (SE)RR spectra, i.e. an increase of the band intensities. The intermediate of the immobilised PpDyP is quite stable, with a half-life of $\sim 15$ min (Fig. 2). With time, the SERR signals decay, without apparent protein denaturation or conversion into Cpd III, most likely due to protein desorption (Fig. 2c).

A comparison of deconvoluted SERR spectra of PpDyP recorded in the absence and presence of $\mathrm{H}_{2} \mathrm{O}_{2}$ reveals a conversion of QS and 


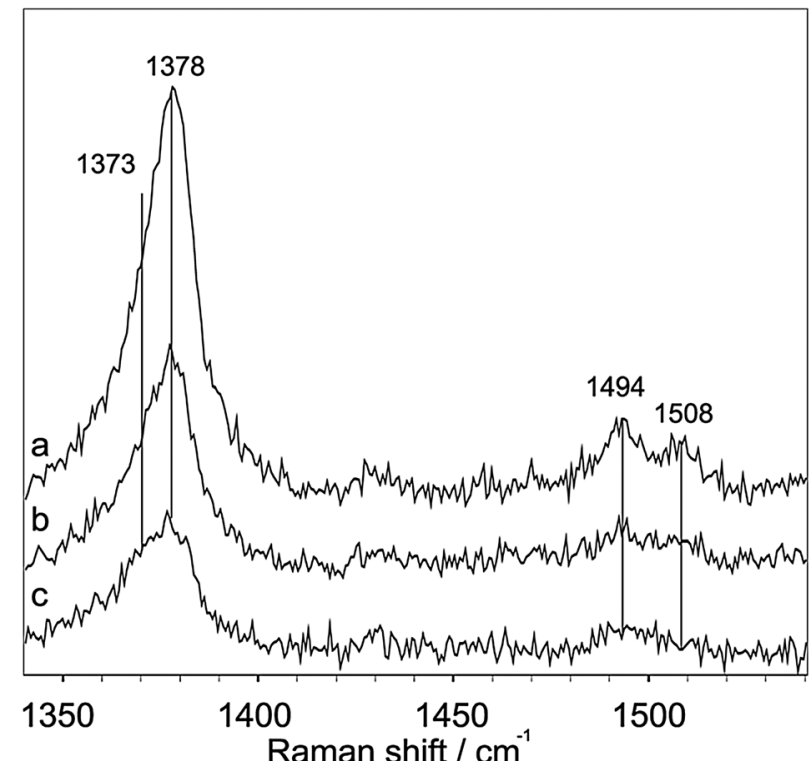

Fig. 2 Time evolution of SERR spectra of Cpd I of immobilised PpDyP. The spectra showing the $\nu_{4}$ and $\nu_{3}$ modes were recorded: 2 (a), 15 (b) and 20 (c) minutes after the addition of $\mathrm{H}_{2} \mathrm{O}_{2}$ to the SERR cell including the PpDyP-loaded Ag support, coated by AOT/MOH. Experimental conditions as in Fig. 1.

HS species into putative Cpd I and HS populations, shedding more light onto disputed physiological relevance of QS species. ${ }^{11,18-20}$ Namely, the origin of this unusual heme configuration, also observed in class III plant peroxidases, cytochromes $c^{\prime}$ and catalase peroxidases (e.g. KatG), is not well understood. ${ }^{11,18-20}$ We have previously demonstrated that it could be observed in B-, but not in A-type DyPs, and that its abundance sensitively depends on the $\mathrm{pH}$, temperature, and physical state of the enzyme. ${ }^{10}$ Here we provide direct experimental evidence that the QS species is capable of reacting with $\mathrm{H}_{2} \mathrm{O}_{2}$ and that it is therefore, most likely catalytically competent in PpDyP.

In conclusion, we presented a vibrational-spectroscopic fingerprint of a catalytic intermediate of the reaction between immobilised PpDyP and $\mathrm{H}_{2} \mathrm{O}_{2}$. To our best knowledge, this is the first report of a catalytic intermediate of a peroxidase obtained by SERR spectroscopy. The technique can therefore be regarded as an important alternative for RR spectroscopic studies of the catalytic cycle of peroxidases ${ }^{11-16,22}$ when fluorescence impedes solution measurements. As a further advantage, SERR requires only small (sub-micromolar) amounts of protein, due to high sensitivity which results from both the plasmonic and resonance enhancements of the signal. In addition, SERR detection of catalytic intermediates is possible at ambient temperature in contrast to EPR spectroscopy that is restricted to cryogenic conditions. Thus, SERR spectroscopy avoids temperaturedependent changes of spin state distributions, ${ }^{9,10,18,19}$ and is applicable to the EPR-silent Cpd II. ${ }^{21}$ As a drawback, SERR spectroscopic identification of intermediates is restricted to species with lifetimes on the minute time scale (e.g. cytochrome $c$ peroxidases and DyPs $)^{1-9,23}$ and can only be used if the enzyme is immobilized under preservation of its native state and function. These conditions are fully satisfied for some DyPs. ${ }^{9}$ Thus, SERR in particular represents a promising approach for a more detailed mechanistic analysis of DyPs from different subfamilies, stabilizing either Cpd I or Cpd II. ${ }^{1-8}$ We have recently observed Cpd I with $t_{1 / 2}>60 \mathrm{~h}$ in distal PpDyP variants D132N and $\mathrm{N} 136 \mathrm{~L}$, in which aspartate 132 was replaced by asparagine and asparagine 136 by leucine, respectively. ${ }^{24}$ Future studies of these systems will contribute to a better understanding of both, molecular determinants of catalytic activity in DyP-type peroxidases, and of their mechanistic properties in the immobilised state, which is a prerequisite for application of these catalytically versatile and efficient enzymes in the construction of bioelectronic devices.

\section{Acknowledgements}

Financial support from DFG (UniCat PH and ST) is acknowledged. We thank S. Mendes for help with protein purification.

\section{Notes and references}

1 X. Liu, Q. Du, Z. Wang, D. Zhu, Y. Huang, N. Li, T. Wei, S. Xu and L. Gu, J. Biol. Chem., 2011, 286, 14922.

2 Y. Sugano, R. Muramatsu, A. Ichiyanagi, T. Sato and M. Shoda, J. Biol. Chem., 2007, 282, 36652.

3 Y. Sugano, Cell. Mol. Life Sci., 2009, 66, 1387.

4 J. N. Roberts, R. Singh, J. C. Grigg, M. E. P. Murphy, T. D. H. Bugg and L. D. Eltis, Biochemistry, 2011, 50, 5108.

5 R. Singh, J. C. Grigg, Z. Armstrong, M. E. P. Murphy and L. D. Eltis, J. Biol. Chem., 2012, 287, 10623.

6 E. van Bloois, D. E. Torres Pazmino, R. T. Winter and M. W. Fraaije, Appl. Microbiol. Biotechnol., 2010, 86, 1419.

7 S. J. Kim and M. Shoda, Appl. Environ. Microbiol., 1999, 65, 1029.

8 A. Santos, S. Mendes, V. Brissos and L. O. Martins, Appl. Microbiol. Biotechnol., 2014, 98, 2053.

9 M. Sezer, T. Genebra, S. Mendes, L. O. Martins and S. Todorovic, Soft Mater., 2012, 8, 10314.

10 M. Sezer, A. Santos, P. Kielb, T. Pinto, L. O. Martins and S. Todorovic, Biochemistry, 2013, 52, 3074.

11 S. M. Kapetanaki, S. Chouchane, S. Yu, R. S. Magliozzo and J. P. M. Schelvis, J. Inorg. Biochem., 2005, 99, 1401.

12 K.-J. Paeng and J. R. Kincaid, J. Am. Chem. Soc., 1988, 119, 7913.

13 V. Palaniappan and J. Terner, J. Biol. Chem., 1989, 264, 16046.

14 W. J. Chaung and H. E. Van Wart, J. Biol. Chem., 1992, 267, 13293.

15 C. M. Reczek, A. J. Sitter and J. Terner, J. Mol. Struct., 1989, 214, 27.

16 S. Hashimoto, J. Teraoka, T. Inubushi, T. Yonetani and T. Kitagawa, J. Biol. Chem., 1986, 261, 11118.

17 F. Siebert and P. Hildebrandt, Vibrational Spectroscopy in Life Sciences, Wiley-VCH Verlag GmbH\&Co. KGaA, Weinheim, 2008.

18 G. Smulevich, A. Feis and B. D. Howes, Acc. Chem. Res., 2005, 38, 433. 
19 B. D. Howes, C. B. Schiodt, K. G. Welinder, M. P. Marzocchi, J.-G. Ma, J. Zhang, J. A. Shelnutt and G. Smulevich, Biophys. J., 1999, 77, 478.

20 M. M. Maltempo, T. H. Moss and M. A. Cusanovich, Biochim. Biophys. Acta, 1974, 342, 290.

21 A. Ivancich, H. M. Jouve, B. Sartor and J. Gaillard, Biochemistry, 1997, 36, 9356.
22 S. Hu and J. R. Kincaid, J. Am. Chem. Soc., 1991, 113, 7189.

23 M. A. Miller, G. W. Han and J. Kraut, Proc. Natl. Acad. Sci. U. S. A., 1994, 91, 11118.

24 S. Mendes, V. Brissos, A. Gabriel, T. Catarino, D. L. Turner, S. Todorovic and L. O. Martins, Arch. Biochem. Biophys, 2015, DOI: 10.1016/j.abb.2015.03.009. 